\title{
Evaluation of Cytotoxic and Genotoxic Effect of Acacia nilotica (L.) Delile Extract Using Allium cepa Bioassay
}

\author{
Samah Bodowara \\ Botany Department, \\ Faculty of Science, \\ Benghazi University, \\ Benghazi - Libya
}

\author{
Fauzia El Garaboli \\ Botany Department, \\ Faculty of Science, \\ Benghazi University, \\ Benghazi - Libya
}

\author{
Salem EI shatshat \\ Botany Department, \\ Faculty of Science, \\ Benghazi University, \\ Benghazi - Libya
}

Article DOI: https://doi.org/10.36713/epra4107

\begin{abstract}
The present study aimed to measure the cytotoxic and genotoxic effect of the bark of A. nilotica extract. Allium cepa assay was used to find out the effect of A. nilotica extract on chromosome structure and behavior during cell division. The root tips meristem cells were treated with different concentration of A. nilotica bark aqueous extract $(0.1,0.01$ and $0.001 \mathrm{mg} / \mathrm{ml}$ ) for 4, 6, 12 and 24 hours, respectively. Cytological analysis revealed decreasing in cell division in all used concentration especially at high ones. The obtained results indicate that aqueous extracts of A. nilotica plant have the ability to decrease the $(M I \%)$ values with increasing the concentration at $(P<0.005)$.

All treatments have caused different kind of mitotic abnormalities and chromosomal aberrations, such as: change percentage of mitotic phases, $C$-mitosis, stickiness, chromosome bridges, Micronucleus and vagrant chromosome. The action of A. nilotica bark extract on the genetic material led to decrease in dividing cells number which was concentration and time depended. This inhibition of cell division was due to disturbances in nucleus as a result of inhibition of DNA synthesis.
\end{abstract}

KEY WORDS: Allium cepa assay: A. nilotica: Chromosomal aberrations; MI.

\section{INTRODUCTION}

Plant natural products derivatives have received considerable attention in recent years due to their diverse pharmacological properties (Govind and Sahni, 2011). Acacia nilotica (L.) Delile (Figure 1), commonly known as babul kikar or Indian gum and Arabic tree in Libya, this plant has been recognized worldwide as a multipurpose tree (National Academy of sciences 1980). It is widely distributed throughout arid and semi- arid zones of the world and can play an important role in soil productivity. It is a relatively fast growing, drought resistant multipurpose legume with the ability of biological nitrogen fixation.(Benso, 2009).

Acacia nilotica (Fabaceae )has been reported to be very useful in treating diarrhea and cough in human. It is reported to have habitual medicine uses such as appetite enhancer, strength and nutrient supplement and clear out circumcision wound
Antioxidant have been implicated in many diseases, malaria, cardiovascular diseases, diabetes and etc. Natural antioxidants such as flavonoids, phenolics, tannis, curcumin and terpenoids are found in $A$. nilotica (Benso, 2009).

Allium cepa bioassay is relatively cheap and given quick results showing the genotoxic effect on plant cells (Fiskesjo, 1985). The chromosome of apical meristem of Allium roots have sensitivity and in dissociable for known the genetic mutagen because of its small number of chromosomes $(2 n=16)$ with large size (Rank \& Nielson, 1994and Fiskesjo, 1985).

A number of studies noted the uses of medicinal plant extracts activity and, many studies looked for their influence on genetic material by using different plant extract and dose (Fiskesjo, 1993). Therefore, The present study was designed to evaluate anti mutagenic activities of aqueous extract of the bark of Acacia nilotica on Allium cepa root tips 
cells to find out the effect on chromosomal structure and behavior during cell division by studying the mitotic index(MI\%) in the treated cells.

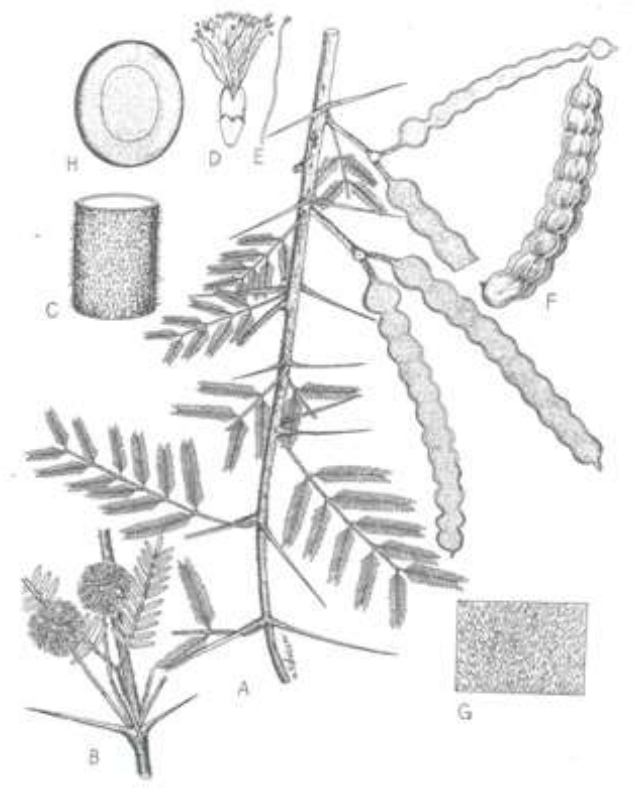

Figure (1) showed the Libyan Acacia nilotica plant (branch, leaves and fruit) from Jafri \& El-Gadi (1978).

\section{MATERIALS AND METHODS}

Collection of plant material: Stem bark of the mature plant of Acacia nilotica was collected from Al-kofra city, Libya which is located in the middle of the Libyan Desert in a particularly isolated area.

Preparation of plant extract: Ethanol extract of the stem bark was prepared with slight modification. 50 gram of sample of the stem bark of the plant was air dried before ground into powder. The blended material was transferred into beaker with $10 \mathrm{ml}$ of $90 \%$ ethanol at ambient temperature $\left(28 \pm 2 \mathrm{C}^{\circ}\right)$ the mixture was extracted by agitation on a rotatory shaker. Extraction was allowed to proceed for $48 \mathrm{~h}$. The mixture was decanted and the solvent was removed by evaporated in active hode at room temperature. Active growing root tip of Allium cepa Treated at Different concentration (0.1-0.01-0.001) of Acacia extract exposed for different times $(4 \mathrm{~h}-6 \mathrm{~h}$ $-12 \mathrm{~h}-24 \mathrm{~h})($ Agrawl et al. 2010$)$

Pre-Treatment: Allium cepa bulbs were grown for 2-3days in distilled water at room temperature with change the water every day when roots reached $2-4 \mathrm{~cm}$ in length, the bulbs were treated with different concentration of Acacia nilotica extracts (Bark) $0,1-0,01-0,001 \mathrm{mg} / \mathrm{ml}$, bulbs of $A$. cepa was growing in water without plant extract were used as control. The solution were changed daily and after $48 \mathrm{~h}$, root tips from each bulbs was harvested, fixed in Carnoy's fixative (95\% ethanol/glacial acetic acid 3:1 $(\mathrm{v} / \mathrm{v})$ for $24 \mathrm{~h}$. And then proceed for slides preparation or stored in $70 \%$ alcohol. The technique of bulbs use is described by (Fiskesjo 1993).
Slide preparation: Preparation of slides was carried out according to Sharma and Sharma (1999) Chromosomes technique. After per - treatment the root tips were washed a few times with distilled water they were hydrolyzed with $1 \mathrm{~N} \mathrm{Hcl}$ at $60-70 \mathrm{c}^{\circ}$ for 9$15 \mathrm{~min}$. After hydrolyzed the roots were washed with $45 \%$ acetic acid. then about $1-2 \mathrm{~cm}$ of the root tips were cut and placed on the slide. A few drops of aceto - orcein was dropped on the root tip and left for 20 min for staining. The root tips were then squashed. The cover slip was carefully lowered on to avoid air bubbles and sealed with clear nail polish.

Observation of specimens: The slides were observed under the light microscope at $400 \mathrm{X}$ mitotic index and chromosome aberration in mitotic phases was counting in minimum of 100 cell per slide and 1000 of the cells for each experiment was studied the experiment was replicated three times with three roots for each mitotic index replicate. The mitotic index was obtained as follows: The number of mitotic cells dividing on to The total number of cells (Grant, 1982).

Statistical Analysis: Statistical program (SPSS) was used to calculate the mean, standard error and other parameters including correlation and one way ANOVA analysis. p-value $<0.005$ was used as significant level of all calculations.

\section{RESULTS}

The results of treatment with extract of $A$. nilotica at different concentrations of $0.1,0.01,0.001$ and control 0.00 for 4, 6, 12 and 24 hours are shown in table1. 
The mean of total number (TC) of analyzed cells included divided and non-divided cells, mean of mitotic cells (MC) and mitotic index (MI) value were recorded. Antimitotic activity of $A$. nilotica was observed in cells treated with high concentration comparing on treated cells at all exposure duration in this studies. A drop in the mitotic activity was evidence in root tip cells treated with 0.1 and 0.01 concentration of $A$. nilotica. the highest value of MI $(10.50,10.60)$ wear observed in cells treated with low concentration $(0.001)$ at 4 and 12 hour treatment. while the lowest value of MI in cells treated was observed with 0.1 concentration for $12 \mathrm{~h}$ exposure time.
Statistical analysis of data in table (1) showed that $A$. nilotica had a significant effect on $\mathrm{MI} \%$ of treated root tips. At $4 \mathrm{~h}$ treatment, there was signify antimitotic effect of $A$. nilotica extract of 0.1 and 0.01 concentration which were 4.99 and 0.89 respectively comparing to the control. There were increases in MI of root tip cells treated with lowest concentration (0.001). At 6h exposure time, all tested plant extract concentrations showed decrease in the rate of cell division which was $6.09,6.03$ and 0,081 for concentrations of $0.1,0,01$ and 0.001 , respectively.

Table (1) Showed the effects of Acacia. nilotica extract on Allium. cepa root tips in $4 \mathrm{~h}, 6 \mathrm{~h}, \mathbf{1 2 h}, \mathbf{2 4 h}$.

\begin{tabular}{|c|c|c|c|c|c|}
\hline Duration & Treatment & T.M.C & M.C & MI\% & AB\% \\
\hline 4h & Control & 558.08 & 53.50 & 9.5900 & 0.00 \\
\hline & 0.1 & 781.25 & 39.00 & 4.9900 & 0.00 \\
\hline & 0.01 & 534.25 & 48.25 & .0890 & 3.2000 \\
\hline & 0.001 & 505.00 & 54.75 & 10.600 & .03000 \\
\hline & Control & 558.08 & 53.50 & 9.5900 & 0.000 \\
\hline & 0.1 & 660.75 & 40.25 & 6.0910 & 0.00 \\
\hline & 0.01 & 469.75 & 29.75 & 6.3300 & 4.12000 \\
\hline & 0.001 & 900.00 & 73.50 & .0810 & 6.0900 \\
\hline & Control & 558.08 & 53.50 & 9.5900 & 0.00 \\
\hline & 0.1 & 800.00 & 12.50 & 1.5600 & 1.2100 \\
\hline & 0.01 & 377.75 & 20.50 & 5.3600 & 1.6100 \\
\hline & 0.001 & 550.00 & 57.75 & 10.500 & 8.0600 \\
\hline & Control & 558.08 & 53.50 & 9.5900 & 0.00 \\
\hline & 0.1 & 418.00 & 9.75 & .0230 & 1.0700 \\
\hline & 0.01 & 537.75 & 9.50 & 1.7600 & 1.0070 \\
\hline & 0.001 & 903.75 & 69.25 & 7.600 & 10.3900 \\
\hline
\end{tabular}

*T.M.C= total mitotic index, M.C= mitotic cells, MI\%= mitotic index. 0.00 no result.

The inhibitory effect of all treated $A$. nilotica extract concentrations was significant comparing to control. There was no significant effect between the inhibitory action of both high concentration 0.1 \& 0.01 .
Changes in MI were very clear in $12 \mathrm{~h}$ exposure time to $A$. nilotica extract. MI values were $1.56,5.36$ and 10.50 at $0.1,0.01$ and 0.001 concentration respectively (figure 2 ). 


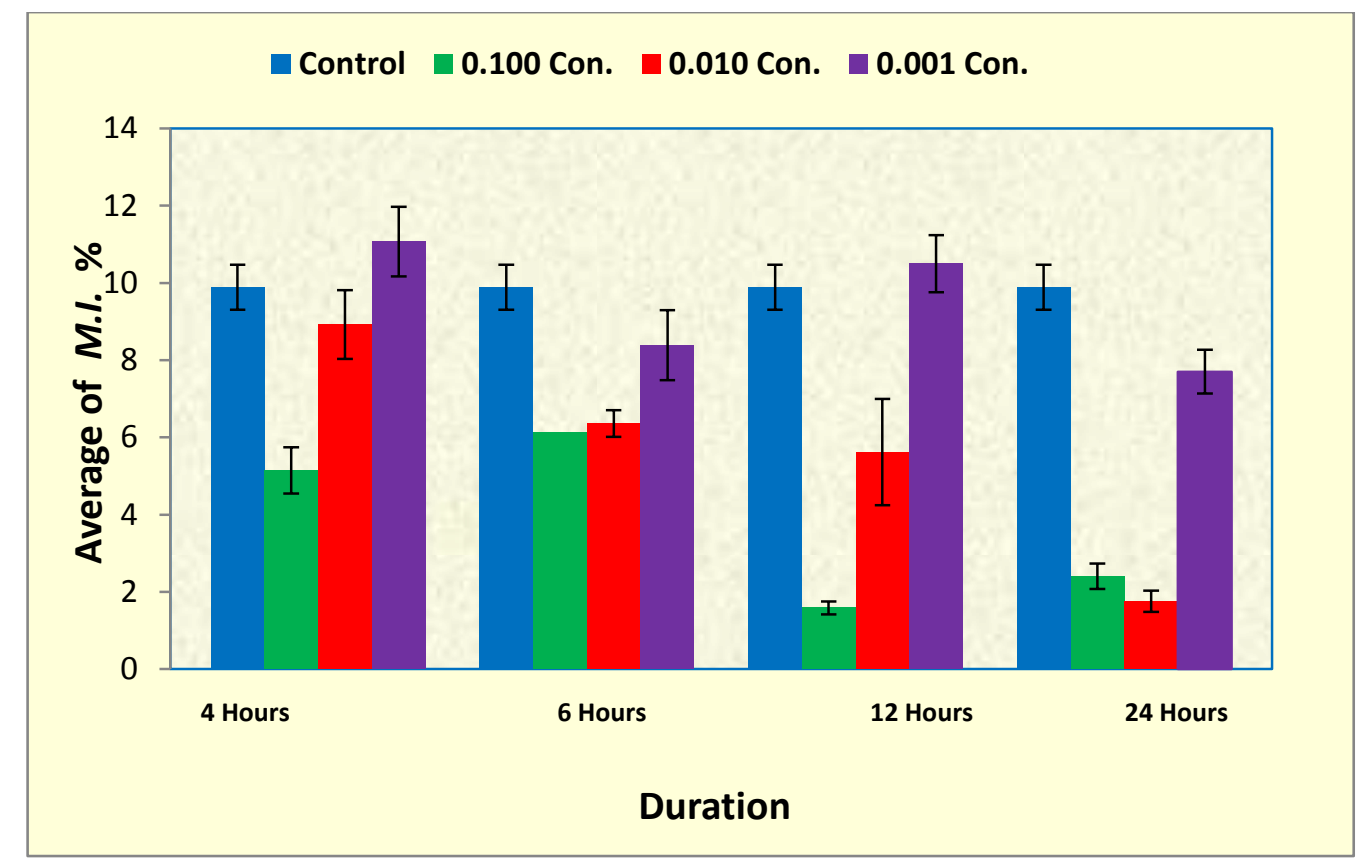

Figure (2); Effect of different concentration of Acacia nilotica on Average of MI\% of Allium cepa root

Induction of cell division abnormality

Abnormalities in divided root tip cells of A. cepa treated cells were carefully studied. figure (3) showed that A. nilotica extract had cytotoxic effect on chromosome behavior during cell division compering to control which lead to cell death at high

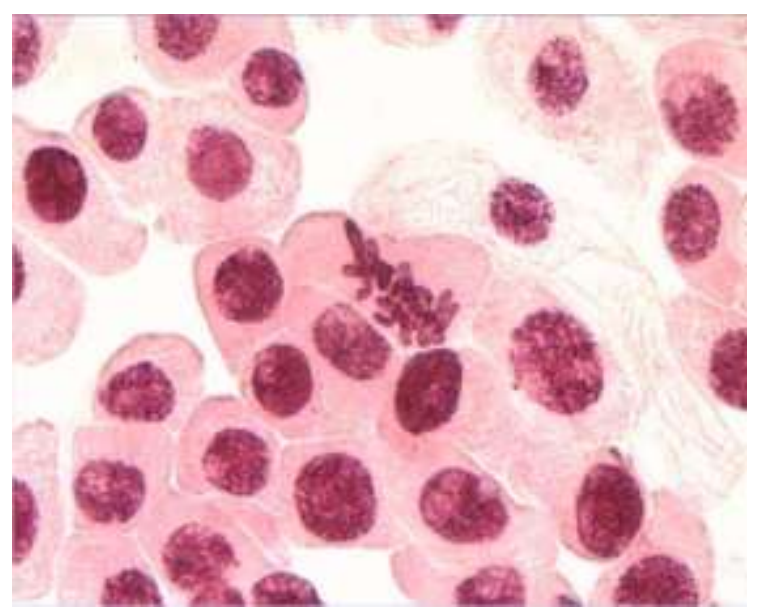

A concentrations of $(0.1,0.01)$ and presence of abnormal divided cells by decrease the plant extract concentration. Some of the physiological aberrations that commonly observed in this study were stickiness, bridges, micronucleus, c-mitosis and vagrant chromosomes (Figure 4).

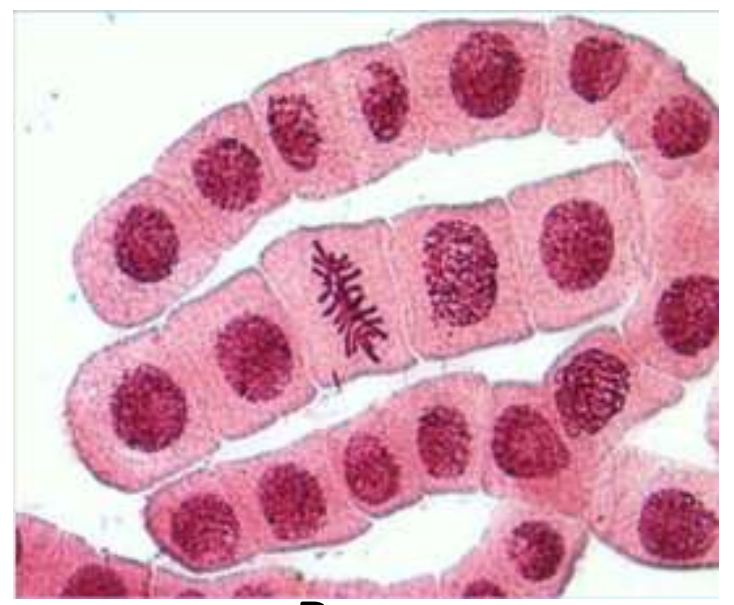

B

Figure (3); Chromosome aberration observed in A. cepa meristimatic cells exposed to ethanol extracts of $A$. nilotica. (A) normal interphase and (B) stickiness. 

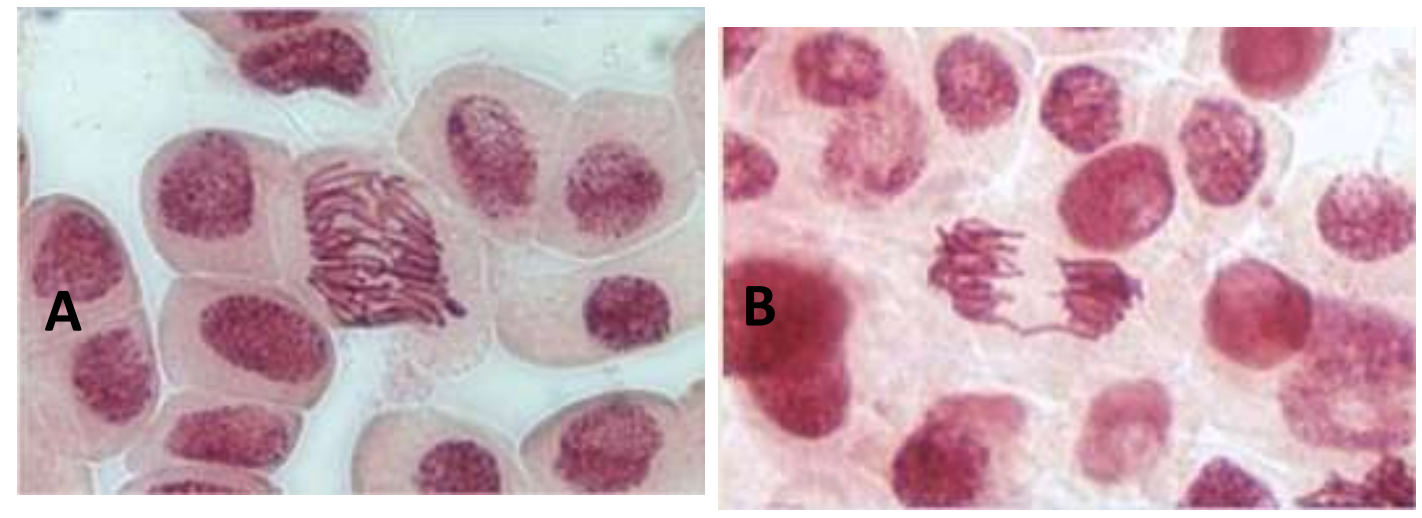

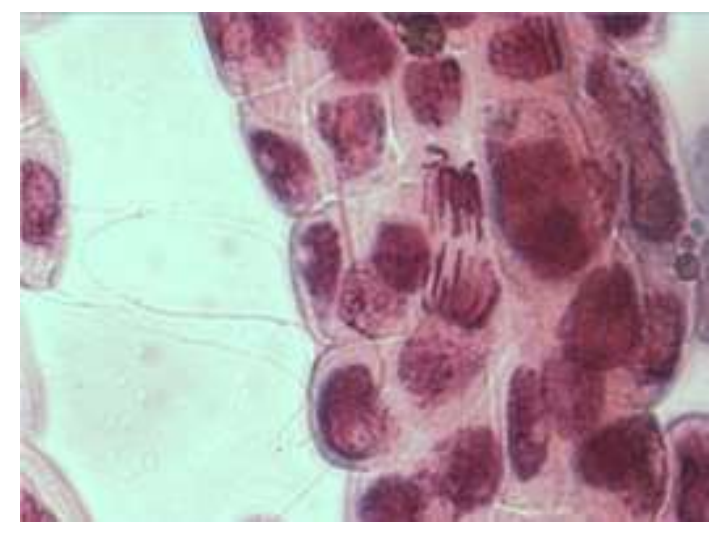

C

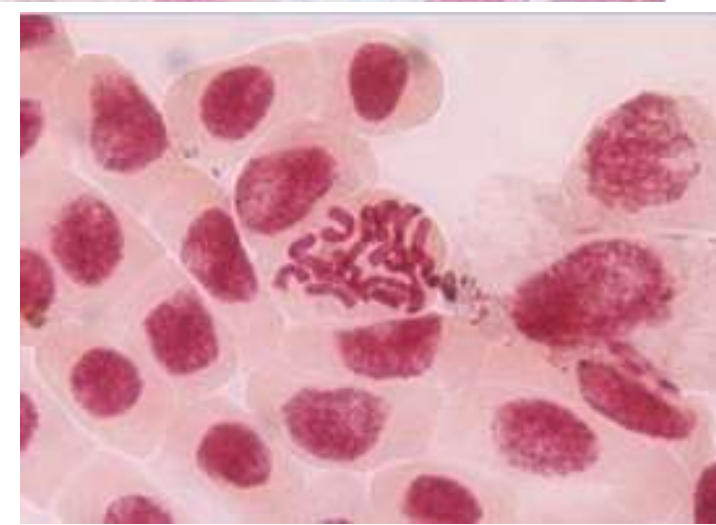

D

Figure (4); Chromosome aberrations observed in $A$. cepa meristematic cells exposed to ethanol extracts of $A$. nilotica (A)normal anaphase, (B) chromosome bridge, (C) vagrant chromosome and (D) c-mitosis.

\section{DISCUSSION}

The abnormalities of chromosomes could be due to the blockage of DNA synthesis or inhibition of spindle formation. Acacia nilotica extract may not even allow the initiation of their biosynthesis. The reduction of the mitotic index might be explained as being due to the obstruction of the onset of prophase, the arrest of one or more mitotic phases or the slowing of the rate of cell progression though mitosis. chromosome aberration provided important information and may be considered an efficient test to investigate the genotoxic potential of the treatments analyzed. The chromosome aberrations observed at all concentrations of the treatment were chromosome stickiness, bridges, micronucleus, c-mitosis and vagrant chromosomes(figure 3 and 4). These aberrations were due to the effect of the extract on the spindle formation and thus resulted in cell division disturbance. Chromosome bridges(figure 4) indicating the clastogenic effect caused by chromosome breaks, whereas vagrant chromosome and c-metaphase increase the risk for aneuploidy.
Some of the physiological aberrations that were commonly observed in this study were stickiness.

A remarkable correlation between the frequencies of stickiness and the bridges was observed. This supports the hypothesis that stickiness may result from improper folding of chromosome fibers which makes the chromatids connected by means of sub chromatid bridge show ever stickiness may be interpreted as a result of deploy merisation of DNA, partial dissolution of nucleoprotein, breakage and exchanges of the basic folded fiber units of chromatids and the stripping of the protein covering of DNA in chromosome According to Fiskesjo (1993), sticky chromosome indicated a highly toxic, irreversible effect, probably leading to cell death.

Another remarkable abnormality was chromosome bridges. Chromosome bridges were commonly observed during anaphase \& telophase. The bridges noticed in the cells were probably formed by breakage and fusion of chromosome or sub chromatid. According to chromosome bridges that may be caused by stickiness of chromosome, which made their separation and free movement complete 
and thus they remained connected by bridges. A low frequency of c-mitosis, vagrant chromosome and micronucleus was also observed. Their presence may be attributed to the failure of the spindle apparatus to organize and function in a normal way (Fiskesjo, 1985). Similar observation have been made by other workers where c-mitosis was regarded as indicative of a weak toxic effect which may be reversed. Micronucleus in root meristem cells of $A$. cepa is manifestation of fragments or vagrant chromosome (Yi, et al; 2003).

Using medicinal plants in medicine is a common method in different countries of the world. From the present study, it appeared that $A$. nilotica ethanol extract, which is used frequently in the traditional medicine, clearly exhibits chromotoxic and mitodepressive effects at $0.001 \mathrm{mg} / \mathrm{ml}$, Therefore, it is necessary to take precautions when using this extract as an alternative traditional remedy healing systems. In addition, further cytogenetic studies dealing with clastogenicity and genotoxicity of this extract with more comprehensive genotoxicity assessment in plant model may reveal further interesting results for its usage for human welfare.

\section{REFERENCES}

1. Agrawal, S., Kulkami, GT. And VN. Sharma (2010). A comparative study on the antioxidant activity of methanol extract of acacia, adv. Nat Appl. Sc., 4(1) :78-84

2. Benso, A. (2009). Phytochemical and antibacterial investigation of bark extracts of Acacia nilotica, journal of medicinal planets research.,3(2):082-085.

3. Fiskesjö, G. (1985).Allium test on river water from Braan and sexan before and after closure of a chemical factory. Ambio, 14(2): 99-103.

4. Fiskesjö, G. (1985). The Allium test as a standard in environmental monitoring. Heraditas. 102, 99112.

5. Fiskesjö, G. (1993). The Allium test in wastewater monitoring. Environmental Toxicology and Water Quality, 8, 291-298.

6. Govind, P. and Sahni, Y. P. (2011). A review on hepato protective activity of silymarin. International Journal in Ayurveda and Pharmacy.2(1):75-79.

7. Grant, W. F. (1978). Chromosome aberration in plant as monitoring System. Environ. Health Perspect.,27:37-43.

8. Grant, W. F.(1982).chromosome aberration assay in Allium report of the U.S. Environmental protection .Agency Gene-Tox program. Mutant. Res. Genet. Toxical. Environ. Mutagen. 99,273291.

9. Rank, j. and M. H. Nilson (1997). Allium cepa anaphase-telophase root tip chromosome aberration assay on N-methyl- $N$ nitrosoureamaleichydrazide, sodium azide, and ethylmethanesulfonate Mutat. Res. Genet. Toxicol. Environ. Mutagen. 390:121-127.
10. Sharma, A.K. and Sharma A. (1999). Chromosome Techniques, Theory and practice. $3 r d$ ed; Butterworth, London.

11. Jafri, S. M. and A. El-Gadi (1978). Libyan flora, Al Faateh university, faculty of science, Department of Botany, Tripoli.

12. Yi, H. and Z. Meng (2003).Genotoxicity of hydrate sulfur dioxide on root tips of Allium sativum and Vicia faba. Mutat. Res. Genet. Toxicol. Environ. Mutagen. 537, 109-114. 